\title{
Rendimiento escolar y condiciones de pobre- za: el rol mediador de las funciones ejecutivas
}

\section{Korzeniowski, C. ${ }^{1}$, Cupani, M. $^{2}$, Ison, $\mathrm{M}^{3}{ }^{3}$ y Difabio, $\mathrm{H}^{4}$.}

1 Instituto de Ciencias Humanas Sociales y Ambientales (INCIHUSACONICET). Centro Científico Tecnológico (CCT Mendoza - CONICET), Argentina.

${ }^{2}$ CIPSI - Grupo Vinculado Centro de Investigaciones y Estudios sobre Cultura y Sociedad (CIECS) - CONICET. Facultad de Psicología, Universidad Nacional de Córdoba, Argentina.

3 Instituto de Ciencias Humanas Sociales y Ambientales (INCIHUSACONICET). Centro Científico Tecnológico (CCT Mendoza - CONICET), Argentina.

${ }^{4}$ Instituto de Investigaciones Cuyo (CIC) - CONICET - Mendoza, Argentina.

\section{Argentina}

Correspondencia: Celina Korzeniowski. Av. Ruiz Leal s/n (CP 5500), Parque General San Martín, Mendoza. Argentina. E-mail: ckorzeniowski@mendoza-conicet.gob.ar

(C) Education \& Psychology I+D+i and Ilustre Colegio Oficial de la Psicología de Andaluacía Oriental (Spain) 


\section{Resumen}

Introducción. El presente trabajo tiene como objetivo analizar si las funciones ejecutivas (FE) predicen el rendimiento escolar (RE) de niños pertenecientes a diferentes estratos socioeconómicos bajos, controlando el efecto de la edad y del nivel socioeconómico (NSE).

Método. La muestra estuvo compuesta por 178 niños argentinos de ambos sexos (52\% varones), de 6 a 10 años de edad, pertenecientes a NSE Bajo Superior (41\%), Bajo Inferior (39\%) y Marginal (20\%). Los niños fueron evaluados con una batería de tests neuropsicológicos de FE y con tests de aprovechamiento escolar.

Resultados. El modelo propuesto explicó el 69\% del RE de los niños. Las FE fueron el predictor directo más significativo y, además, mediaron las relaciones entre NSE y RE.

Conclusión. El empobrecimiento de las condiciones materiales y socioculturales de la familia, se asocia con un menor desempeño de las FE en los niños, lo que repercute negativamente en su RE.

Palabras Clave: rendimiento escolar, funciones ejecutivas, nivel socioeconómico, pobreza, niños argentinos. 


\section{Abstract}

Introduction. This study aims at analysing whether EF may predict the SP of children from different low socioeconomic strata, having controlled the effects of age and socioeconomic status (SES).

Method. The sample was made up of 178 Argentine children of both genders (52\% boys), between 6 and 10 years of age, belonging to the upper-low SES (41\%), lower-low SES (39 $\%)$ and marginal SES (20\%). The children were evaluated by means of a battery of neuropsychological EF and school achievement tests.

Results. The proposed model accounted for $69 \%$ of the SP of the children. EFs were the most significant direct predictor and, in addition, mediated the relationship between SES and SP.

Conclusion. In line with previous findings, these results indicate that the impoverishment of family material and sociocultural conditions is associated with a lower EF performance among children, which negatively impacts their SP.

Keywords: school performance, executive functions, socioeconomic status, poverty, Argentine children 


\section{Introducción}

El rendimiento escolar (RE) es un proceso complejo condicionado por diversos factores de índole personal y contextual. Un tema de constante preocupación en torno al RE es la persistente brecha educativa entre niños socio-económicamente favorecidos y desfavorecidos, la cual juega un rol central en la transmisión intergeneracional de la pobreza (Crook y Evans, 2014; Fitzpatrick, McKinnon, Blair y Willoughby, 2014; Hackman, Farah y Meaney, 2010). La probabilidad de no ir a la escuela es cuatro veces mayor entre los niños más pobres del mundo que entre los más ricos, y cinco veces mayor la de no terminar la enseñanza primaria sin alcanzar las competencias básicas (Organización de las Naciones Unidas para la Educación, la Ciencia y la Cultura [UNESCO], 2015). América Latina es una de las regiones con mayores índices de desigualdad educativa en el mundo: los niños y niñas pobres que viven en zonas rurales tienen mayor probabilidad que sus pares urbanos provenientes de hogares ricos de repetir un grado y abandonar la educación primaria (Oficina Regional de Educación de la UNESCO para América Latina y el Caribe [OREALC/UNESCO], 2015).

Esta situación se agrava cuando se percibe la existencia de diferentes circuitos de escolarización conforme a los orígenes sociales de los estudiantes, dando como resultado un menor rendimiento escolar en niños de estratos socioeconómicos bajos en comparación con sus pares de estratos medios o altos (Dirección Nacional de Información y Evaluación de la Calidad Educativa [DiNIECE], 2013; Enríquez, 2011; Krüger, 2013; OREALC/UNESCO, 2015). Estas inequidades educacionales emergen tempranamente en la infancia y se robustecen a medida que el niño transita la escolaridad primaria y secundaria, conllevando a menores logros educativos y menores ingresos en la adultez (Blair y Raver, 2014; Diamond y Lee, 2011). En consecuencia, resulta de gran importancia comprender cómo las condiciones de pobreza generan en los niños un riesgo académico temprano y de largo alcance (Fitzpatrick et al., 2014).

Una posible vía de estudio es analizar los efectos de la pobreza sobre el desarrollo cognitivo, específicamente, sobre las funciones ejecutivas (FE), dado que se consideran uno de los sistemas cognitivos más sensibles a la influencia ambiental (Hackman et al., 2010; Noble, MacCandliss y Farah, 2007). Las FE involucran un conjunto de capacidades cognitivas de alto orden que controlan y regulan los comportamientos, emociones y cogniciones necesarios 
para alcanzar metas y resolver problemas (Diamond, 2013). Diferentes estudios dan evidencia de que las FE constituyen uno de los factores predictores más significativos del RE desde la etapa preescolar hasta la adultez (Best, Miller y Naglieri, 2011; Checa y Rueda, 2011; Diamond, 2013; Welsh et al., 2010). Esto se debe en gran medida a que un adecuado desarrollo de las FE contribuye a que los niños puedan mantenerse enfocados en la información relevante de la tarea, controlar los distractores, planificar, organizar y monitorear el proceso de aprendizaje, formular estrategias para el logro de un objetivo, detectar errores, evaluar diferentes soluciones a problemas y reflexionar sobre pensamientos y acciones (Blair y Raver, 2014).

Durante la etapa escolar, los niños realizan importantes avances en las FE y en las competencias escolares de manera simultánea, sugiriéndose la superposición de procesos de desarrollo (Fuhs, Nesbitt, Farran y Dong, 2014). En efecto, en los primeros grados escolares se registra un pico en la fuerza de las correlaciones entre FE y RE (Best et al., 2011; Welsh et al., 2010). En consecuencia, el intenso desarrollo de las capacidades de control cognitivo que se informa entre los 6 y 8 años y entre los 10 y 12 años de edad (Flores-Lázaro, CastilloPreciado y Jiménez-Miramonte, 2014; Hughes, 2011), podría considerarse como un posible factor que facilite el aprendizaje y el desempeño del niño en clase.

Las relaciones entre FE y RE, sin embargo, resultan atravesadas por múltiples factores, uno de ellos es la calidad de la estimulación cognitiva recibida por el niño en sus primeros años de vida. Se ha informado que los niños en riesgo social por pobreza presentan un menor desempeño en atención, memoria de trabajo, planificación, control inhibitorio, fluidez verbal, flexibilidad cognitiva, organización, metacognición y monitoreo (Arán Filippetti y Richaud de Minzi, 2012; Hackman et al., 2010; Ison, Greco, Korzeniowski y Morelato, 2015; Lipina et al., 2011; Musso, 2010; Noble et al., 2007). Esta alteración del desarrollo cognitivo se asocia con problemas de conducta, fracaso escolar, relaciones sociales conflictivas, afectando los aprendizajes infantiles y pronosticando un menor nivel educativo (Diamond y Lee, 2011).

Esto se debe en gran medida a que el ambiente de crianza de los niños socioeconómicamente desfavorecidos suele estar caracterizado por situaciones de estrés crónico y la ausencia de experiencias estimulantes que promuevan el desarrollo de las FE (Fitzpatrick et al., 2014). Se ha documentado que los padres de menor nivel educativo leen poco a sus hijos, 
dialogan menos con ellos, emplean un discurso de menor complejidad y un vocabulario más constreñido en las interacciones, lo que se asocia con menores recursos lingüísticos y cognitivos en los infantes (Ardila, Rosselli, Matute y Guajardo, 2005; Hoff, 2003). La conjunción de estos factores disminuye la calidad de la estimulación cognitiva, a lo que se suma una menor disponibilidad en sus hogares de recursos materiales y herramientas para promover el aprendizaje (Bradley y Corwyn, 2002).

En suma, la pobreza impacta el rendimiento escolar a través de múltiples vías, pudiendo ser las FE un factor mediador de dicho impacto. En efecto, algunos estudios (Fitzpatrick et al., 2014; Nesbitt, Baker-Ward y Willoughby, 2013) con preescolares estadounidenses han revelado que las capacidades de control cognitivo en los niños mediaron las relaciones entre nivel socioeconómico (NSE) y el desempeño escolar en tareas matemáticas y de lectura. Otro estudio realizado con niños estadounidenses de diferentes etnias observó que el NSE de los hogares medido cuando los niños eran pequeños ( 1 a 24 meses de edad) predijo sus habilidades matemáticas y de lectura en grados posteriores ( $5^{\circ}$ grado), y que esta relación estuvo mediada por las habilidades de planificación (Crook y Evans, 2014). Por lo tanto, en base a los resultados previos y tomando en consideración que a medida que las condiciones de pobreza se acentúan, el riesgo académico de los niños se incrementa, el presente trabajo se propone como objetivo analizar si las FE median las relaciones entre los gradientes de pobreza y el rendimiento escolar en niños argentinos de 6 a 10 años de edad.

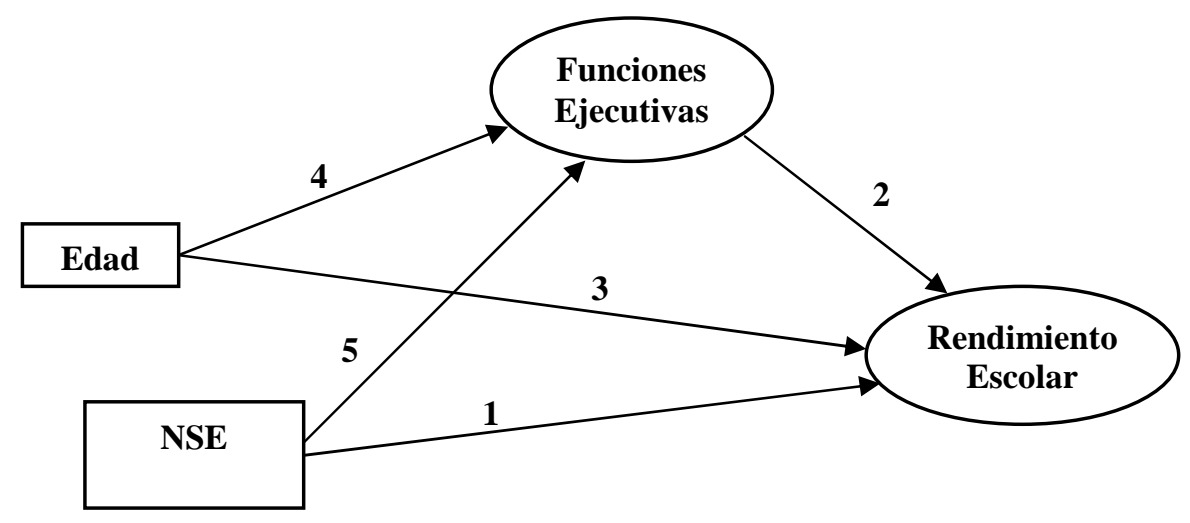

Figura 1. Predictores del rendimiento escolar en niños. Nota: NSE informa gradientes de pobreza. 
En la Figura 1, se presenta gráficamente un modelo explicativo del rendimiento académico. De acuerdo con los estudios previos (i.e. Checa y Rueda, 2011; Crook y Evans, 2014; Davidson, Amsoa, Anderson y Diamond, 2006; Diamond, 2013; Fuhs et al., 2014; Hackman et al., 2010; Lipina et al., 2011; Noble et al., 2007; DiNIECE, 2013; Welsh et al., 2010), se propone que el NSE (path 1), las FE (path 2) y la Edad (path3) tienen una incidencia directa y positiva sobre el RE. Por otro lado, se considera que las FE modulan el RE de escolares primarios, luego de controlar el efecto de la edad (path 4) y la pertenencia de los niños a diferentes estratos socioeconómicos (path 5).

\section{Objetivos e hipótesis}

Los objetivos específicos del trabajo fueron:

1. Analizar si las funciones ejecutivas modulan el rendimiento escolar de niños argentinos, luego de controlar el efecto de la edad y su pertenencia a diferentes estratos socioeconómicos.

2. Explorar si las funciones ejecutivas median las relaciones entre gradientes de pobreza y rendimiento escolar en los niños participantes.

Las hipótesis del trabajo se desprenden del modelo teórico propuesto (Figura 1) y fueron las siguientes:

1. El rendimiento escolar es modulado por la edad de los niños, el nivel socioeconómico familiar y las funciones ejecutivas.

2. Las funciones ejecutivas explican parcialmente las diferencias socioeconómicas del rendimiento escolar de los niños.

\section{Método}

\section{Participantes}

La muestra empleada fue no probabilística intencional y estuvo compuesta por $178 \mathrm{ni}$ ños argentinos de ambos sexos (52\% varones), de 6 a 10 años de edad (M =7,24, DE =1,17), pertenecientes a NSE Bajo Superior (41\%), Bajo Inferior (39\%) y Marginal (20\%). Los niños 
cursaban de $1^{\circ}$ a $3^{\circ}$ grado de educación primaria en dos escuelas de gestión pública urbanomarginadas de Mendoza (Argentina). Se consideró como criterio de inclusión que los niños hubiesen sido autorizados por sus padres o tutores, mediante consentimiento escrito, a participar del proyecto. Se excluyeron del estudio aquellos niños que presentaron: a) problemas neurológicos, psicológicos o psiquiátricos, b) trastornos de aprendizaje previamente diagnosticados y c) dos o más años de extraedad escolar.

\section{Instrumentos}

Escala Magallanes de Atención Visual (EMAV, García-Pérez y Magaz-Lago, 2000). Es un test visual de ejecución continua que consiste en reconocer figuras iguales a un modelo propuesto entre un conjunto de figuras diferentes. Evalúa atención focalizada, atención sostenida y eficacia atencional. Este instrumento ha sido adaptado para escolares argentinos de 6 a 12 años de edad (Carrada, 2011). El índice de confiabilidad estimado por el método de partición por mitades fue elevado ( $r h o=0,89$ ). Para la muestra en estudio el índice de confiabilidad fue satisfactorio $(r h o=0,87)$.

Laberintos de Porteus (Porteus, 2006). Este test mide habilidades de planificación y control inhibitorio. Está integrado por 10 laberintos ordenados en una secuencia de dificultad creciente. Los participantes deben resolver la tarea teniendo en cuenta tres reglas: no levantar el lápiz durante el trazado del laberinto, no atravesar líneas y no ingresar a callejones sin salida. El TLP presenta una consistencia interna moderadamente alta $(\alpha=0,80$, Krikorian y Bartok, 1998). En este trabajo se utilizó el Índice de Calidad de Porteus (Marino, Fernández y Alderete, 2001) para valorar las habilidades de planificación, y la adaptación de la puntuación Q (Korzeniowski, 2015) para valorar la capacidad de control inhibitorio. En esta muestra, los índices de consistencia interna para las puntuaciones de planificación $(\alpha=0,81)$ fueron satisfactorios. De la misma manera, la confiabilidad inter-examinadores para los nueve ítems que componen la puntuación Q fue adecuada (Coeficiente de Correlación Intraclase [CCI] oscilando entre 0,79 a 0,99$)$.

Formación de Conceptos - Batería III COG Woodcock-Muñoz (Muñoz-Sandoval, Woodcock, Mc Grez y Mather, 2005). Esta prueba permite valorar razonamiento categorial y flexibilidad cognitiva. Es de administración individual y se toma desde los 5 años hasta la adultez. Este test consiste en una tarea de aprendizaje controlado en la cual el sujeto debe 
identificar una regla o concepto en un conjunto de estímulos presentados visualmente. La confiabilidad de este instrumento para la franja etaria de 5 a 19 años es de $r h o=0,94$ (MuñozSandoval et al., 2005). Para la muestra en estudio se obtuvo una confiabilidad adecuada (rho $=0,80)$.

Entrevista metacognitiva para niños (Lucangeli y Cornoldi, 1997). Es una entrevista semidirigida que consta de 8 preguntas, 4 abiertas y 4 con formato Likert que permite valorar el conocimiento meta-estratégico del niño en relación con una tarea de categorización y flexibilidad cognitiva. Está dividida en dos partes, la primera valora las habilidades de predicción y planificación, por lo cual se administra antes de que el sujeto realice la tarea propuesta, y la segunda mide habilidades de monitoreo y evaluación, y se administra inmediatamente después de que el sujeto ha finalizado la tarea. Para las preguntas abiertas se estimó el grado de acuerdo entre examinadores (CCI entre 0,86 a 0,95) y para los cuatros ítems Likert se estimó la consistencia interna $(\alpha=0,52)$.

Batería de Aprovechamiento Woodcock-Muñoz (Woodcock y Muñoz- Sandoval, 1996). En este trabajo se administraron tres de los sub-test que conforman la Batería. Identificación de Letras-Palabras. Mide las habilidades lectoras del sujeto para identificar una letra o palabra con precisión y rapidez. Los ítems están ordenados en forma de dificultad creciente: vocales, consonantes, palabras frecuentes e inusuales. La prueba es aplicable desde los 3 a los 80 años de edad y presenta una consistencia interna promedio elevada ( $r h o=0,92)$. Para la muestra en estudio, se obtuvo un coeficiente de confiabilidad excelente (rho $=0,98)$. Dictado. El test es administrado como una prueba tradicional de dictado y mide habilidades básicas de escritura (dibujo de letras, deletreo, puntuación y ortografía). Se aplica individualmente desde los 3 años y en adultos. Los ítems están ordenados en una secuencia de dificultad creciente. La confiabilidad de este instrumento es de $r h o=0,91$. Para la muestra en estudio se obtuvo un valor de rho $=0,92$. Problemas Aplicados. Mide la habilidad del sujeto para analizar y resolver problemas prácticos en Matemática. Consta de 59 problemas verbales con soporte gráfico o escrito, ordenados en una secuencia de dificultad creciente. Esta prueba se administra individualmente desde los 3 años y en adultos. La confiabilidad es de $r h o=0,91$. Para la muestra en estudio se obtuvo una confiabilidad adecuada $(r h o=0,81)$. 
Nivel socioeconómico (Comisión de Enlace Institucional, 2006). Este índice permite valorar la posición socioeconómica de un hogar a través de variables indirectas, excluyendo el nivel de ingresos como un criterio. Considera dos variables centrales: inserción laboral y nivel educativo del principal sostén del hogar (PSH), y otras variables secundarias, como acceso a sistemas de salud. En este trabajo sólo se consideraron las dos variables centrales para estimar el NSE familiar.

\section{Procedimiento}

Se solicitó el aval de la Dirección General de Escuelas de la provincia de Mendoza como también la autorización escrita de los directivos de las escuelas participantes. Se requirió el consentimiento escrito a los padres de los alumnos para la participación de sus hijos. Por otra parte, se explicó a los niños autorizados las características de las tareas por realizar, se los invitó a participar voluntariamente y se les informó sus derechos como participantes. Las evaluaciones fueron realizadas por la autora principal en cuatro sesiones de 30 minutos cada una. Para la misma se contó con un aula ventilada, luminosa y dispuesta por la escuela. En la primera sesión se administró la EMAV en forma grupal. En las tres sesiones restantes se administraron en forma individual los tests de FE y de aprovechamiento escolar. El procedimiento se desarrolló bajo normas éticas internacionales (American Psychological Association, 2002) y contó con la aprobación del comité de ética del Instituto de Ciencias Humanas, Sociales y Ambientales, dependiente de CONICET-Argentina.

\section{Análisis de Datos}

Tres pasos se realizaron con el fin de preparar los datos para los análisis propuestos. En primer lugar, se evaluó el patrón de valores perdidos para estimar si el mismo respondía a una distribución aleatoria, mediante la rutina de Análisis de los Valores Perdidos del SPSS 19. En un segundo paso, se identificaron casos atípicos univariados mediante el cálculo de puntuaciones estándar para cada una de las variables. Se consideraron atípicos aquellos casos con puntuaciones $z$ superiores a 3,29 (prueba de dos colas, $p<0,001$ ). Previo a un posible descarte se realizó la prueba de distancia de Mahalanobis con $p<0,001$ con la finalidad de detectar casos atípicos multivariados (Tabachnick y Fidell, 2001). En un tercer paso, para comprobar los supuestos de normalidad de la muestra se realizaron análisis de asimetría y curtosis en cada variable. Como criterio para evaluar los índices de asimetría y curtosis se consideró como excelente valores entre $+1,00$ y $-1,00$, y adecuados valores inferiores a $+2,00$ y $-2,00$ 
(George y Mallery, 2011). Un último análisis consistió en el diagnóstico de multicolinealidad a los fines de estimar la existencia de variables altamente correlacionadas o redundantes $(r \geq$ $0,90)$.

Para evaluar el Modelo de Ecuaciones Estructurales se utilizó el software AMOS 19.0 (Arbuckle y Wothke, 1999) y el método de estimación empleado fue el de Máxima Probabilidad. Para evaluar el ajuste de los modelos se utilizó el estadístico chi-cuadrado, la razón de chi-cuadrado sobre los grados de libertad (CMIN/DF), el índice de ajuste comparativo (CFI), el índice de bondad de ajuste (GFI), el error cuadrático medio de aproximación (RMSEA) y el error cuadrático medio estandarizado (SRMR). Para este trabajo, la bondad de ajuste se consideró con los siguientes criterios: la razón de chi-cuadrado sobre los grados de libertad con valores inferiores a 3,0 (Kline, 2011); los índices CFI y GFI con valores entre 0,90 y 0,95 o superiores (considerados como ajuste aceptable a excelente) y, finalmente, para el caso del RMSEA y SRMR valores entre 0,05 y 0,08 (Hu y Bentler, 1995). Por último, los efectos indirectos y totales de las variables del modelo fueron analizados mediante el método de remuestreo bootstrap (Efron, 1979). Distintas investigaciones de simulación de datos (MacKinnon, Lockwoo y Williams, 2004) han demostrado que este procedimiento presenta un control más riguroso del Error Tipo I, y que es recomendable utilizar este método en vez de la prueba de Sobel. Para aplicar este método se utilizó el enfoque bootstrap paramétrico de Monte Carlo con una estimación de los intervalos de confianza corregidos al 95\% (BC, bias corrected), y se generaron 1.000 muestras elegidas al azar desde los datos.

\section{Resultados}

\section{Preparación de los datos}

Los casos perdidos no superaron el 5\% por lo cual se decidió imputarlos mediante el método Estimación-Maximación. Posteriormente, se descartaron nueve casos atípicos univariados $(4,8 \%)$, quedando una muestra de 178 niños. Los índices de asimetría y curtosis estuvieron comprendidos entre $-2,00$ y $+2,00$ considerados adecuados para los análisis paramétricos propuestos (George y Mallery, 2011). El análisis de la multicolinealidad arrojó una correlación elevada de 0,89 entre dos pruebas de aprovechamiento mientras que el resto de las correlaciones fueron moderadas. En la Tabla 1 se presentan las intercorrelaciones de las variables de interés en los escolares participantes. 
Tabla 1. Estadísticos descriptivos y correlaciones bivariadas entre variables cognitivas, de desempeño escolar, edad y NSE en los escolares $(n=178)$

\begin{tabular}{|c|c|c|c|c|c|c|c|c|c|c|c|c|}
\hline & $M$ & $D E$ & 1 & 2 & 3 & 4 & 5 & 6 & 7 & 8 & 9 & 10 \\
\hline 1. Eficacia atencional & 0,25 & 0,14 & & & & & & & & & & \\
\hline 2. Flexibilidad cognitiva & 13,11 & 4,82 & $0,35 * *$ & - & & & & & & & & \\
\hline 3. Planificación & 4,26 & 1,93 & $0,41 * *$ & $0,45^{* *}$ & - & & & & & & & \\
\hline 4. Control Inhibitorio & 8,07 & 4,09 & $-0,22 * *$ & $-0,14$ & $-0,21 * *$ & - & & & & & & \\
\hline 5. Metacognición & 6,80 & 2,63 & 0,13 & $0,52 * *$ & $0,24 * *$ & $-0,02$ & - & & & & & \\
\hline 6. Identificación Palabras & 23,00 & 15,4 & $0,53 * *$ & $0,41 * *$ & $0,40 * *$ & $-0,30 * *$ & $0,19^{*}$ & - & & & & \\
\hline 7. Problemas aplicados & 19,79 & 3,72 & $0,55^{* *}$ & $0,59 * *$ & $0,37 * *$ & $-0,20 * *$ & $0,33 *$ & $0,67 * *$ & - & & & \\
\hline 8. Dictado & 17,55 & 7,31 & $0,51 * *$ & $0,42 * *$ & $0,39 * *$ & $-0,27 * *$ & $0,22 * *$ & $0,89 * *$ & $0,70 * *$ & - & & \\
\hline 9. Edad & 7,24 & 1,17 & $0,43^{* *}$ & $0,19 * *$ & $0,36^{* *}$ & $-0,24 * *$ & 0,03 & $0,60 * *$ & $0,52 * *$ & $0,59 * *$ & - & \\
\hline 10. NSE & & & 0,08 & $0,21^{* *}$ & 0,11 & $-0,16^{*}$ & 0,12 & 0,09 & 0,07 & 0,13 & 0,04 & - \\
\hline
\end{tabular}

Nota: $* * p<0,01$ (bilateral). * $p<0,05$ (bilateral). NSE $=$ nivel socioeconómico. ${ }^{\text {a }}$ Las correlaciones entre NSE y las demás variables se calcularon con el estadístico Rho de Spearman.

\section{Evaluación del Modelo Propuesto}

Para estimar el modelo de ecuaciones estructurales se utilizó una estrategia de modelado en dos etapas (Kline, 2011). Primero se evaluó el modelo de medida para examinar la estructura latente que subyace a las distintas medidas y, luego, se evaluó el modelo de estructura para observar el ajuste y la varianza entre las variables.

Modelo de Medida. Se evaluó un modelo de medida compuesto por dos variables latentes y 8 indicadores como variables observables con sus respectivos errores de medición. La cantidad de indicadores por factor latente osciló entre 3 y 5 . Los estadísticos obtenidos indicaron que el modelo no se ajustó de forma adecuada a los datos $(\mathrm{GFI}=0,89, \mathrm{CFI}=0,88$; RMSEA $=0,14)$. Un examen de los índices de modificación permitió observar un aparente solapamiento de contenidos entre un par de indicadores (flexibilidad y metacognición). Por lo tanto, se consideró incluir en el modelo el parámetro de covarianza entre los errores y el modelo resultó aceptable $(\mathrm{CFI}=0,93, \mathrm{GFI}=0,94, \mathrm{RMSEA}=0,11)$. Los pesos de regresión estandarizados $(p \leq 0,05)$ en el factor Funciones Ejecutivas oscilaron entre 0,28 y 0,68 y en el factor Rendimiento Escolar entre 0,75 a 0,94. 
Modelo de Estructura. El modelo de estructura se presenta en la Figura 2, en él se pueden apreciar los coeficientes path estandarizados y los coeficientes de determinación $\left(R^{2}\right)$. Los resultados indican que el modelo se ajustó adecuadamente a los datos $\left(X^{2}{ }_{(31,178)}=83,43\right.$, $p<0,001, \mathrm{CFI}=0,93, \mathrm{GFI}=0,93, \mathrm{RMSEA}=0,09$ y CMIN/DF $=2,69)$ y explicó un 69\% del rendimiento escolar.

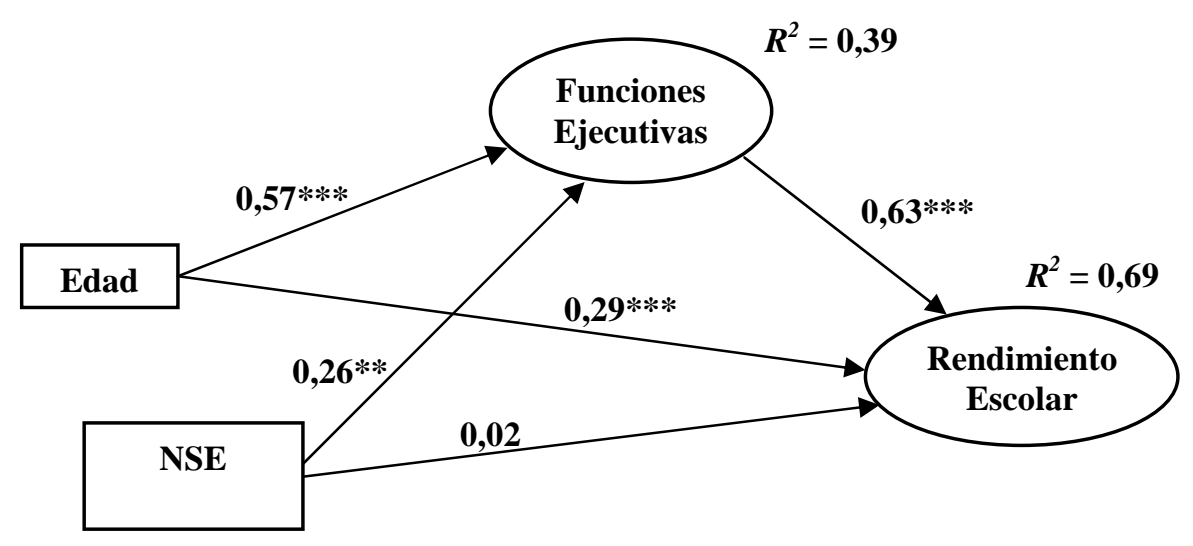

Figura 2. Modelo de ecuaciones estructurales para testear el impacto de la edad, las condiciones de pobreza y las funciones ejecutivas en el rendimiento escolar en niños. $* * * p<0,001, * * p<0,01$.

Para determinar si los datos corroboran las relaciones propuestas se analizaron los efectos directos, indirectos y totales. Tal como se propuso (path 2 y 3 ), las variables FE $(\beta=$ $0,63)$ y edad $(\beta=0,26)$ presentaron un coeficiente path estandarizado estadísticamente significativo y en dirección positiva sobre rendimiento académico. Contrario a lo expuesto (path 1), el NSE $(\beta=0,02)$ no realizó una contribución directa y significativa sobre el rendimiento. En relación a la variable FE, el modelo explicó el 39\% de su varianza. Tal como se propuso en el modelo (path 4 y 5$)$, la edad $(\beta=0,57)$ y el NSE $(\beta=0,26)$ presentaron un coeficiente path estandarizado estadísticamente significativo y en dirección positiva sobre FE, indicando que a medida que los niños aumentaban en edad o pertenecían a un estrato socioeconómico menos pobre sus FE se incrementaban. 


\section{Análisis de los efectos indirectos y totales}

Se registró que las FE mediaron de manera significativa la relación entre edad y RE ( $\beta$ $=0,36$ ), indicando que a medida que los niños aumentaban en edad incrementaban sus capacidades de control cognitivo, lo que se asoció con un aumento significativo de sus competencias escolares. El efecto total de la variable edad sobre $\operatorname{RE}(\beta=0,62)$ fue estadísticamente significativo. Por último, se observó que las FE mediaron de manera significativa la relación entre NSE y RE $(\beta=0,16)$, señalando que a medida que los niños pertenecían a estratos socioeconómicos menos pobres, sus FE aumentaban, y de manera consecuente, su desempeño escolar. En suma, los resultados hallados señalan que las capacidades de control cognitivo fueron el predictor directo más significativo del RE en los niños participantes y que, además, mediaron las relaciones entre condiciones de pobreza y RE, así como entre edad y RE.

\section{Discusión}

La pobreza impacta el rendimiento escolar por múltiples vías, una de ellas es a través del desarrollo cognitivo infantil. El presente estudio realizado con escolares primarios argentinos provee evidencia a este postulado. Los resultados obtenidos informan que las capacidades de control cognitivo predijeron el RE en niños de estratos socioeconómicos bajos, tras controlar el efecto de la edad y el NSE. Estos datos robustecen y extienden trabajos previos que han corroborado el rol crítico de las FE en el desempeño escolar (Best et al., 2011; Diamond, 2013; Welsh et al., 2010).

Una diferencia importante entre este estudio y los anteriores es haber integrado en el constructo FE un conjunto de indicadores escasamente utilizados, tales como planificación y metacognición. Usualmente, la variable latente FE se conforma con memoria de trabajo, control inhibitorio/atencional y flexibilidad (Nesbitt et al., 2013). Sin embargo, las demandas cognitivas se incrementan a medida que el niño progresa en la trayectoria escolar, requiriendo cada vez mayores capacidades para planificar sus tareas, resolver problemas y monitorear sus procesos de pensamiento y acciones; de allí la importancia de enriquecer la variable latente con FE complejas. 
Otra diferencia de interés es haber encontrado evidencia sobre el rol mediador de las FE en la relación condiciones de pobreza y desempeño escolar, en una muestra de niños latinoamericanos. Estudios previos han testeado estas relaciones comparando niños estadounidenses de estratos socioeconómicos bajos y altos (Crook y Evans, 2014; Fitzpatrick et al., 2014; Nesbitt et al., 2013). Los resultados informados en este trabajo cobran mayor relevancia si se tiene en cuenta que el NSE no moduló de manera directa el RE de los escolares, lo que se encuentra en línea con estudios previos que han informado que el NSE evaluado como constructo no suele ser sensible para predecir diferencias en el desempeño escolar (Crook y Evans, 2014; Nesbitt et al., 2013). No obstante, en este trabajo se observó que las condiciones socioculturales del hogar modularon el desempeño cognitivo de los niños, y a través de esta vía impactaron sobre las competencias escolares de los niños.

Otro aspecto de interés es haber incorporado en el modelo la variable edad para controlar su efecto en el desempeño cognitivo y escolar de los niños. Por una parte, los resultados señalaron que a medida que los niños aumentaban en edad, su rendimiento escolar se incrementaba significativamente. Si bien este dato está acorde con el carácter graduado de la educación en Argentina, resulta un aporte interesante porque contradice aquellos postulados que sostienen un efecto acumulativo de las pobrezas (Krüger, 2013) en detrimento del rendimiento escolar de los niños en riesgo social. En este estudio, los niños mayores presentaron competencias de lectura, escritura y resolución de problemas superiores en comparación con los niños menores. Estos datos son alentadores y brindan apoyo a trabajos previos que consideran a la escuela urbano-marginada como una institución que puede generar prácticas y condiciones que disminuyan las desventajas socioculturales de origen y promuevan un aprendizaje de calidad (Enríquez, 2011).

Por otra parte, los resultados indicaron que el desarrollo evolutivo de las FE es un factor que contribuye a incrementar el dominio de los niños en competencias escolares, en correspondencia con investigaciones previas que informan la importancia de fortalecer e incrementar las FE como un modo de favorecer el aprendizaje escolar (Best et al., 2011; Blair y Raver, 2014; Diamond y Lee, 2011; Welsh et al., 2011).

Sin embargo, este trabajo no está libre de limitaciones. Una de ellas está referida a que la medida de NSE empleada no tuvo valor predictivo sobre el desempeño escolar de los niños 
participantes. Aunque es probable que esta dificultad se asocie con una menor variabilidad de la muestra, en tanto los participantes pertenecían a una población socialmente vulnerable, podría formularse una explicación alternativa: la escasa capacidad del índice utilizado para capturar las diferencias socioculturales vinculadas con los diferentes estratos de pobreza. Quizás hubiese sido necesario contemplar factores tales como hacinamiento, percepción de subsidio estatal, disponibilidad de recursos materiales y servicios, capital cultural, acceso a sistemas de salud. En consecuencia, se sugiere que estudios futuros utilicen un constructo de NSE que posibilite identificar con mayor exactitud las variaciones socioculturales de los hogares más pobres que modulan el RE.

Una segunda limitación refiere a no haber empleado un diseño longitudinal ni haber incorporado en el modelo variables tales como CI, velocidad de procesamiento, desarrollo del lenguaje, diseño curricular, informadas en estudios previos como predictores significativos del RE (Arán Filippetti y Richaud de Minzi, 2012; Blair y Raver, 2014; Crook y Evans, 2014; Nesbitt et al., 2013). En nuevas investigaciones sería de importancia testear los resultados hallados en este estudio, incorporando las variables controles mencionadas. Finalmente, los resultados que se informan requieren ser contextualizados en tanto provienen de escolares primarios de zonas socialmente vulnerables de Argentina, por lo cual no pueden ser generalizados a niños de otras regiones o niveles socioeconómicos. Resultaría de interés probar el modelo propuesto en diferentes poblaciones latinoamericanas con mayor heterogeneidad socioeconómica.

En suma, este estudio brinda continuidad a trabajos recientes que informan que las FE explican en parte las diferencias en el desempeño escolar a causa del efecto de las disparidades socioculturales del ambiente familiar (Crook y Evans, 2014; Fitzpatrick et al., 2014; Nesbitt et al., 2013). Su principal aporte es haber observado un patrón de relaciones transitivas entre gradientes de pobreza, FE y RE. A nuestro conocimiento, este es uno de los primeros trabajos que ha inspeccionado dichas relaciones en una muestra de escolares primarios argentinos.

\section{Conclusión}

Las condiciones de pobreza generan un riesgo académico temprano y de largo alcance en los niños. Por ello, identificar las características infantiles que pueden contribuir a reducir- 
lo, es un eslabón necesario para aminorar la persistente brecha económica y sociocultural asociada con la indigencia. El presente trabajo incrementa evidencia previa que señala a las FE como un sistema cognitivo sensible a la experiencia ambiental, en tanto documenta que las FE explicaron parcialmente las diferencias en el RE de los niños asociadas a las diferentes configuraciones socioculturales de los estratos socioeconómicos más pobres. Estos resultados denuncian la importancia de implementar programas de estimulación cognitiva o diseños curriculares orientados a fortalecer las capacidades de control cognitivo en los niños socioeconómicamente desfavorecidos, con miras a promover un desempeño escolar exitoso. 


\section{Referencias}

American Psychological Association. (2002). Ethical principles of psychologists and code of conduct. American Psychologist, 57, 1060-1073. Recuperado de http://www.apa.org/ethics/code/principles.pdf

Arán Filippetti, V., y Richaud de Minzi, M.C. (2012). A structural analysis of executive functions and socioeconomic status in school-age children: Cognitive factors as effect mediators. The Journal of Genetic Psychology, 173(4), 393-416. doi:10.1080/00221325.2011.602374

Arbuckle, J., y Wothke, W. (1999). AMOS users guide, version 5.0. Chicago: Small Waters.

Ardila, A., Rosselli, M., Matute, E, y Guajardo, S. (2005). The Influence of the Parents' Educational Level on the Development of Executive Functions. Developmental Neuropsychology, 28(1), 539-560. doi:10.1207/s15326942dn2801_5

Best, J., Miller, P., y Naglieri, J. (2011). Relations between Executive Function and Academic Achievement from Ages 5 to 17 in a Large, Representative National Sample. Learn Individ Differ, 21(4), 327-336. doi: 10.1016/j.lindif.2011.01.007

Blair, C., y Raver, C. C. (2014). Closing the Achievement Gap through Modification of Neurocognitive and Neuroendocrine Function: Results from a Cluster Randomized Controlled Trial of an Innovative Approach to the Education of Children in Kindergarten. PLOS ONE, 9(11), 1-13. doi:10.1371/journal.pone.0112393

Bradley, R. H., y Corwyn, R. (2002). Socioeconomic status and child development. Annual Review of Psychology, 53, 371-399. doi:10.1146/annurev.psych.53.100901.135233

Carrada, M. (2011). El mecanismo atencional en niños escolarizados: Baremación de instrumentos para su medición. (Tesis doctoral). Universidad Nacional de San Luis, Argentina.

Checa, P., y Rueda, M. R. (2011). Behavioral and Brain Measures of Executive Attention and School Competence in Late Childhood. Developmental Neuropsychology, 36(8), 1018-1032. doi:10.1080/87565641.2011.591857

Comisión de Enlace Institucional. (2006). NSE 2006 Informe final. Recuperado de: https://es.scribd.com/doc/64283742/Nivel-Socio-Economico-2006-de-Argentina-2311-2006-Informe-Final-SAIMO-AAM-CEIM

Crook, S. R., y Evans, G. W. (2014). The Role of Planning Skills in the Income-Achievement Gap. Child Development, 85(2), 405-411. doi:10.1111/cdev.12129 
Davidson, M., Amsoa, D., Anderson, L. C., \& Diamond, A. (2006). Development of cognitive control and executive functions from 4 to 13 years: Evidence from manipulations of memory, inhibition, and task switching. Neuropsychology, 44, 2037-2078. doi: 10.1016/j.neuropsychologia.2006.02.006

Diamond, A. (2013). Executive functions. Annual Review of Psychology, 64(1), 135-168. doi:10.1146/annurev-psych-113011-143750

Diamond, A., y Lee, K. (2011). Interventions Shown to Aid Executive Function Development in Children 4 to 12 Years Old. Science, 333, 959-964. doi:10.1126/science.1204529

Dirección Nacional de Información y Evaluación de la Calidad Educativa. (2013). Operativo Nacional de Evaluación 2013. Informe nacional de resultados muestra $3^{o}$ y $6^{o}$ año de Educación primaria. Recuperado de http://portales.educacion.gov.ar/diniece/

Enríquez, P. (2011). El espacio urbano como lugar de marginalidad social y educativa. Argonautas, $1,48-79$.

Efron, B. (1979). Bootstrap methods: another look at the jackknife. Annals of Statistics, 7, 126. doi:10.1214/aos/1176344552

Fitzpatrick, C., McKinnon, R. D., Blair, C., y Willoughby, M. (2014). Do preschool executive function skills explain the school readiness gap between advantaged and disadvantaged children? Learning and Instruction, 30, 25-31. doi:10.1016/j.learninstruc.2013.11.003

Flores-Lázaro, J.C., Castillo-Preciado, R. E., y Jiménez-Miramonte, N. A. (2014). Desarrollo de funciones ejecutivas, de la niñez a la juventud. Anales de Psicología, 30(2), 463473. doi:10.6018/analesps.30.2.155471

Fuhs, M., Nesbitt, K., Farran, D., y Dong, N. (2014). Longitudinal Associations Between Executive Functioning and Academic Skills Across Content Areas. Developmental Psychology, 50(6), 1698-1709. doi:10.1037/a0036633.

García-Pérez, M., y Magaz-Lago, A. (2000). Escala Magallanes de Atención Visual. Madrid: Albor-cohs.

George, D., y Mallery, P. (2011). IBM SPSS Statistics 21 step by step: A simple guide and reference (13th ed.). Boston: Pearson Education.

Hackman, D. A., Farah, M. J., y Meaney, M. J. (2010). Socioeconomic status and the brain: mechanistic insights from human and animal research. Neuroscience, 11, 651-659. doi: $10.1038 / \mathrm{nrn} 2897$ 
Hoff, E. (2003). The specificity of environmental influence: Socioeconomic status affects early vocabulary development via maternal speech. Child Development, 74(5), 13681378. doi:10.1111/1467-8624.00612

Hu, L., y Bentler, P. (1995). Evaluating model fit. In: R. Hoyle (Ed.), Structural equation modelling: Concepts, issues and applications (pp. 76-99). Thousand Oaks, CA: Sage Publications.

Hughes, C. (2011). Changes and Challenges in 20 Years of Research into the Development of Executive Functions. Infant and Child Development, 20, 251-271. doi:10.1002/icd.736

Ison, M., Greco, C., Korzeniowski, C. y Morelato, G. (2015). Selective Attention: a Comparative Study on Argentine Students from Different Socioeconomic Contexts. Electronic Journal of Research in Educational Psychology, 13(2), 343-368. doi: 10.14204/ejrep.36.14092

Kline, R. B. (2011). Principles and practice of structural equation modeling (3a. ed.). New York: Guilford.

Korzeniowski, C. (2015). Programa de estimulación de las funciones ejecutivas y su incidencia en el rendimiento escolar en alumnos mendocinos de escuelas primarias de zonas urbano-marginadas. (Tesis doctoral en Psicología). Universidad Nacional de San Luis, Argentina.

Krikorian, R., y Bartok, J. A. (1998). Developmental data for Porteus Maze Test. The Clinical Neuropsychologist, 12(3), 305-310. doi: 10.1076/clin.12.3.305.1984

Krüger, N. (2013). Segregación Social y Desigualdad de Logros Educativos en Argentina. $\begin{array}{lllll}\text { Archivos Analíticos de Política Educativa, 21(86), 1-26. } & \text { de }\end{array}$ doi:10.14507/epaa.v21n86.2013

Lipina, S. J., Hermida, M. J., Segretin, M. S., Prats, L. Fracchia, C., y Colombo, J. A. (2011). Investigación en pobreza infantil desde perspectivas neurocognitivas. En S.J. Lipina y M. Sigman (Eds), La pizarra de Babel. Puentes entre neurociencia, psicología y educación (pp. 243-264). Buenos Aires: Libros Del Zorzal.

Lucangeli, D., y Cornoldi, C. (1997). Mathematics and Metacognition: What is the nature of the relationship? Mathematical cognition, 3(2), 121-139. doi:10.1080/135467997387443

MacKinnon, D. P., Lockwood, C. M. y Williams, J. (2004). Confidence limits for the indirect effect: Distribution of the product and resampling methods. Multivariate Behavioral Research, 39, 99-128. doi: 10.1207/s15327906mbr3901_4 
Marino, J. C., Fernández, A. L., y Alderete, A. M. (2001). Valores normativos y validez conceptual del test Laberintos de Porteus en una muestra de adultos argentinos. Revista de Neurología Argentina, 26(3), 102-107.

Muñoz-Sandoval, A. F., Woodcock, R. W., Mc Grez, K. S., y Mather, N. (2005). Batería III Woodcock-Muñoz. Itsaca, IL: Riverside Publishing.

Musso, M. (2010). Funciones ejecutivas: un estudio de los efectos de la pobreza sobre el desempeño ejecutivo. Interdisciplinaria, 27(1), 95-110.

Nesbitt, K. T., Baker-Ward, L., y Willoughby, M. L. (2013). Executive function mediates socio-economic and racial differences in early academic achievement. Early Childhood Research Quarterly, 28, 774-783. doi:10.1016/j.ecresq.2013.07.005

Noble, K., McCandliss, B., y Farah, M. (2007). Socioeconomic gradients predict individual differences in neurocognitive abilities. Developmental Science, 10(4), 464-480. doi: 10.1111/j.1467-7687.2007.00600.x

Oficina Regional de Educación de la UNESCO para América Latina y el Caribe. (2015). Informe de Resultados: Factores Asociados. Tercer Estudio Regional Comparativo y Explicativo. Resumen Ejecutivo. Recuperado de http://www.unesco.org/new/es/santiago/terce/

Organización de las Naciones Unidas para la Educación, la Ciencia y la Cultura. (2015). La Educación para Todos, 2000-2015: logros y desafíos. Recuperado de http://es.unesco.org/gem-report/reports

Porteus, S.D. (2006). Laberintos de Porteus (4a ed.). Madrid: TEA Ediciones.

Tabachnick, B. G., y Fidell, L.S. (2001). Using multivariate statistics (4a ed.) Boston: Allyn and Bacon.

Welsh, J. A., Nix, R. L., Blair, C., Bierman, L., y Nelson, K.E. (2010). The Development of Cognitive Skills and Gains in Academic School Readiness for Children from LowIncome Families. Journal of Educational Psychology, 102(1), 43-53. doi: $10.1037 / \mathrm{a} 0016738$

Woodcock, R. W., y Muñoz-Sandoval, A. F. (1996). Batería Woodcock-Muñoz: Pruebas de aprovechamiento-Revisada. Itasca, IL: Riverside Publishing. 